\title{
A topological characterization of holomorphic parabolic germs in the plane
}

\author{
by \\ Frédéric Le Roux (Orsay)
}

\begin{abstract}
J.-M. Gambaudo and É. Pécou introduced the "linking property" in the study of the dynamics of germs of planar homeomorphisms in order to provide a new proof of Naishul's theorem. In this paper we prove that the negation of the Gambaudo-Pécou property characterizes the topological dynamics of holomorphic parabolic germs. As a consequence, a rotation set for germs of surface homeomorphisms around a fixed point can be defined, and it turns out to be non-trivial except for countably many conjugacy classes.
\end{abstract}

1. Introduction. Let $\mathcal{H}^{+}$be the set of orientation preserving homeomorphisms of the plane that fix 0 , and let $h \in \mathcal{H}^{+}$. We are interested in the dynamics of the germ of $h$ at 0 . Imagine one wants to evaluate the "amount of rotation" in a neighbourhood $V$ of 0 by looking at the way the orbit of some point $x \in V$ rotates around 0 . Then two kinds of difficulties can arise:

- if the orbit of $x$ leaves $V$ after a small number of iterations, then the behaviour of $x$ is not significant with respect to the local dynamics;

- if the orbit of $x$ tends to the fixed point 0 , then the rotation of $x$ around 0 is not significant either, because it is not invariant under a continuous change of coordinates.

These difficulties have led Gambaudo and Pécou to introduce the "linking property" (see [GP, Pé]) which demands that inside each neighbourhood of 0 there exist arbitrarily long segments of orbits starting and ending not too close to 0 . In this paper we prove that the only germs that do not share the linking property are the contraction, dilatation and holomorphic parabolic germs. To be more precise, let us define the short trip property, which is the negation of the Gambaudo-Pécou property, as follows.

Definition 1. Let $f \in \mathcal{H}^{+}$. We say that $f$ has the short trip property if there exists a neighbourhood $V$ of the fixed point 0 such that for every

2000 Mathematics Subject Classification: 37E30, 37E45, 37F99.

Key words and phrases: fixed point, rotation set, Leau-Fatou theorem. 
neighbourhood $W$ of 0 , there exists an integer $N_{W}>0$ such that for every segment of orbit $\left(x, f(x), \ldots, f^{n}(x)\right)$ which is included in $V$, and whose endpoints $x, f^{n}(x)$ are outside $W$, the length $n$ is less than $N_{W}$.

Two homeomorphisms $f_{1}, f_{2} \in \mathcal{H}^{+}$are said to be locally topologically conjugate if there exists a homeomorphism $\varphi \in \mathcal{H}^{+}$such that $f_{2}=\varphi f_{1} \varphi^{-1}$ on some neighbourhood of 0 . We are interested in the local dynamics near the fixed point 0 , thus we consider maps up to local conjugacy. Note that any local homeomorphism locally coincides with a homeomorphism defined on the whole plane, so that working with globally defined homeomorphisms is just a matter of convenience and does not alter the results (see [Ham] or [LR1, chapitre 2]). As a consequence, to prove that two homeomorphisms are locally topologically conjugate it suffices to construct the conjugacy on a neighbourhood of 0 .

Definition 2. Let $f \in \mathcal{H}^{+}$, and identify the plane with the complex plane $\mathbb{C}$. We say that $f$ is a locally holomorphic parabolic homeomorphism (or just parabolic) if $f$ is holomorphic on some neighbourhood of $0, f^{\prime}(0)$ is a root of unity, and for every positive $n$ the map $f^{n}$ is not locally equal to the identity.

Note that the hypothesis on $f^{\prime}(0)$ amounts to saying that the differential of $f$ is a rational rotation, and then the last hypothesis is equivalent to saying that $f$ is not locally topologically conjugate to its differential. According to Camacho's version of the Leau-Fatou theorem, if $f \in \mathcal{H}^{+}$is parabolic, then $f$ is locally topologically conjugate to some map

$$
z \mapsto e^{2 i \pi p / q} z\left(1+z^{q r}\right) \quad \text { with } p / q \in \mathbb{Q}, q, r \geq 1 \text {. }
$$

See [Cam], and Figure 1.

We can now state our theorem.

THEOREM 3. Let $f$ be an orientation preserving homeomorphism of the plane that fixes the point 0 . Then $f$ has the short trip property if and only if it is locally topologically conjugate to the contraction $z \mapsto \frac{1}{2} z$, to the dilatation $z \mapsto 2 z$, or to a locally holomorphic parabolic homeomorphism.

As a consequence there are only countably many conjugacy classes failing to have the Gambaudo-Pécou property.

In order to explain where Theorem 3 comes from, let us first discuss the Naishul theorem. In [GP] it was shown that the Gambaudo-Pécou property holds when $f$ preserves area, and then this property is used to prove the Naishul theorem: among area preserving homeomorphisms fixing 0 that are differentiable at 0 and whose differential is a rotation, the angle of the rotation is invariant under a local topological conjugacy. Then the following nice generalization of the Naishul theorem is given by Gambaudo, Le Calvez and 


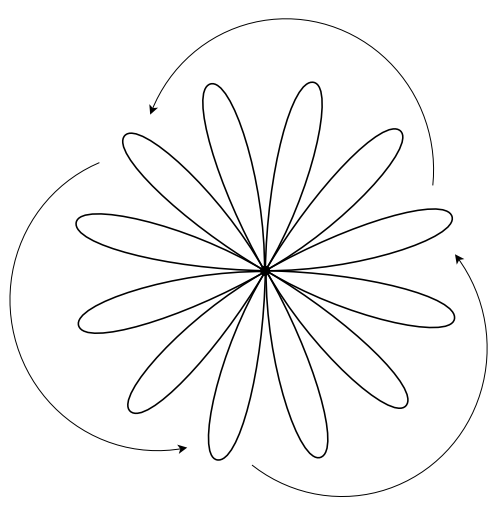

$q r$ periodic petals for $f$

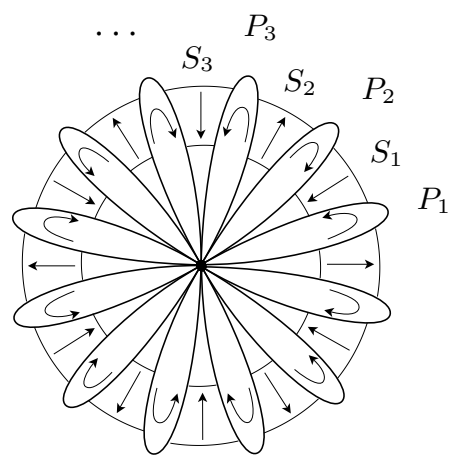

action of $f^{q}$

Fig. 1. Local topological dynamics of $f: z \mapsto e^{2 i \pi p / q} z\left(1+z^{q r}\right)$; here $q=3, p=1, r=2$, so that there are two orbits of attracting petals and two orbits of repulsive petals.

Pécou in [GLP]. As a generalization of differentiability at 0 , they consider the homeomorphisms $f$ for which the fixed point can be "blown up", i.e. replaced by an ideal circle in such a way that $f$ can be extended to a circle homeomorphism (see the precise definition in [GLP]). They prove that for such homeomorphisms, the Poincaré rotation number of the extended circle homeomorphism is invariant under a local topological conjugacy, unless $f$ is a contraction or a dilatation. The strategy of their proof is the following. If $f$ has the Gambaudo-Pécou property, then one can use the arguments in [GP]. Now assume that $f$ is indifferent, that is, $f$ admits arbitrarily small non-trivial invariant compact connected sets $K$ containing 0 ; then one can use Carathéodory's prime ends theory to associate a circle homeomorphism $f_{K}$ to each such $K$, and use the rotation number of $f_{K}$ to prove the topological invariance. Then one proves a last lemma asserting that a germ which is not indifferent and does not have the Gambaudo-Pécou property must be a contraction or a dilatation.

As a consequence of the Leau-Fatou theorem, parabolic maps are indifferent. Thus Theorem 3 can be seen as a generalization of this last lemma. Furthermore, it provides an alternative proof of the generalized Naishul theorem, avoiding the use of prime ends, as follows: we keep the arguments in [GP] to tackle homeomorphisms with the Gambaudo-Pécou property; then, in view of Theorem 3, it only remains to deal with parabolic homeomorphisms, for which the proof is easy because the local dynamics is fully understood.

More generally, in [LR2] we will define a local rotation set for any homeomorphism $f$ in $\mathcal{H}^{+}$. This set is a subset of the extended line $\mathbb{R} \cup\{ \pm \infty\}$, modulo integer translation, and it is a local topological conjugacy invariant. 
Then Theorem 3 will entail that the local rotation set is non-void as soon as $f$ does not fall into the countably many conjugacy classes described by the theorem.

One can also think of Theorem 3 as a local analogue of previous results showing that a simple dynamical property can imply a strong rigidity. The most striking result here is probably the Hiraide-Lewowicz theorem: an expansive homeomorphism on a compact surface is conjugate to a pseudoAnosov homeomorphism (see [Hi, Le]). Closer to our setting, Kerékjártó has shown that an orientation preserving homeomorphism of a closed orientable surface whose singular set is totally disconnected is topologically conjugate to a conformal transformation (see [BK, Ke34a, Ke34b]). Thus, for instance, an orientation preserving homeomorphism $f$ of the plane is conjugate to a translation if and only if it has no fixed point and the family $\left(f^{n}\right)_{n \geq 0}$ is equicontinuous at each point for the spherical metric.

In some sense, Theorem 3 highlights that it is easy to be locally conjugate to a locally parabolic homeomorphism: a homeomorphism that "looks like" a parabolic map will be conjugate to it. In contrast, the examples given in [BLR] reveal how difficult it is to be conjugate to the saddle homeomorphism $(2 x, y / 2)$, and in particular that it is not enough to preserve the hyperbolic foliation. A topological characterization can be given, but it must take into account the sophisticated oscillating set (see, in [BLR], the remark on Fig. 3 as well as part III).

2. Dynamics of parabolic germs. Propositions 12 and 13 below provide a first (classical) characterization of parabolic germs in terms of attracting and repulsive sectors and invariant petals.

2.1. Contractions and attracting sectors. We begin by characterizing the dynamics of contractions. Then we describe attracting sectors. Of course, similar results hold for dilatations and repulsive sectors, although we will not state them explicitly.

Let $f \in \mathcal{H}^{+}$. We will say that a sequence $\left(E_{n}\right)_{n \geq 0}$ of subsets of the plane converges to 0 if for every neighbourhood $W$ of 0 , all but finitely many terms of the sequence are included in $W$. The following result is very classical.

Proposition 4. Let $f \in \mathcal{H}^{+}$. Let $D$ be a topological closed disc $\left(^{1}\right)$ which is a neighbourhood of 0 , and suppose that the orbit $\left(f^{n}(D)\right)_{n \geq 0}$ converges to 0. Then $f$ is locally topologically conjugate to the contraction $z \mapsto \frac{1}{2} z$.

Proof. By hypothesis there exists $n>0$ such that $f^{n}(D) \subset \operatorname{Int}(D)$. Choose some decreasing finite sequence of topological closed discs $D_{i}$ with $D_{0}=D, \operatorname{Int}\left(D_{i}\right) \supset D_{i+1}$, and $\operatorname{Int}\left(D_{n-1}\right) \supset f^{n}\left(D_{0}\right)$. Consider the set

$\left({ }^{1}\right)$ A topological closed disc is a set homeomorphic to the closed unit disc. 


$$
O=\operatorname{Int}\left(D_{n-1}\right) \cap \operatorname{Int}\left(f\left(D_{n-2}\right)\right) \cap \cdots \cap \operatorname{Int}\left(f^{n-1}\left(D_{0}\right)\right) .
$$

Let $U$ be the connected component of $O$ containing the fixed point 0 . The hypotheses on the $D_{i}$ 's entail that $\operatorname{Clos}(f(O)) \subset O$. Since $\operatorname{Clos}(f(U))$ is connected and contains 0, we deduce that $\operatorname{Clos}(f(U)) \subset U$. Furthermore, according to a theorem of Kerékjártó, the set $D^{\prime}=\operatorname{Clos}(U)$ is a closed topological disc (see $[\mathrm{Ke} 23, \mathrm{LCY}]$ ). This disc satisfies $f\left(D^{\prime}\right) \subset \operatorname{Int}\left(D^{\prime}\right)$.

Now the annulus $D^{\prime} \backslash \operatorname{Int}\left(f\left(D^{\prime}\right)\right)$ is a "fundamental domain" for $f$, and can be used to construct a local topological conjugacy between $f$ and the contraction.

We will say that two sets $S$ and $S^{\prime}$ coincide in a neighbourhood of 0 , or have the same germ at 0 , and we will write $S \stackrel{0}{=} S^{\prime}$, if there exists a neighbourhood $V$ of 0 such that $S \cap V=S^{\prime} \cap V$.

Definition 5 (see Figure 2). An attracting sector is a topological closed disc $S$ whose boundary contains 0 , which coincides in a neighbourhood of 0 with its image $f(S)$, and whose orbit $\left(f^{n}(S)\right)_{n \geq 0}$ converges to 0 . The attracting sector is said to be nice if $f(S) \subset S$ and $S \backslash f(S)$ is connected. A (nice) repulsive sector is a (nice) attracting sector for $f^{-1}$.

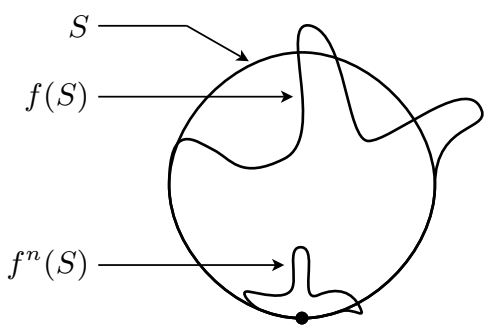

0

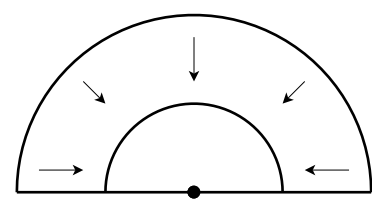

0

Fig. 2. Attracting and nice attracting sectors

\section{Claim 6.}

1. If $S$ is an attracting sector then there exists a nice attracting sector $S^{\prime}$, included in $S$, and having the same germ as $S$ at 0 .

2. If $S^{\prime}$ is a nice attracting sector for $f$, then there exists a homeomorphism $\Phi$ between $S^{\prime}$ and the half-disc $S_{0}=\{|z| \leq 1, y \geq 0\}$ such that the conjugacy relation $\Phi f=\frac{1}{2} \Phi$ between $f$ and the contraction $z \mapsto \frac{1}{2} z$ holds on $S^{\prime}$.

REMARK 7 . Here are some easy consequences of item 2 of the claim.

1. The sets $\Phi^{-1}([-1,0]), \Phi^{-1}([0,1])$ are called the sides of the nice attracting sector; they do not depend on the choice of $\Phi$. 
2. There exist arbitrarily small nice attracting sectors within $S^{\prime}$; moreover, any pair of points $x, y$ on both sides of $S^{\prime}$ are the endpoints of the sides of some nice attracting sector included in $S^{\prime}$.

3. Any homeomorphism $\Phi$ between the union of the sides of $S^{\prime}$ and the segment $[-1,1]$, satisfying the conjugacy relation $\Phi f=\frac{1}{2} \Phi$, can be extended to a homeomorphism between $S^{\prime}$ and $S_{0}$ conjugating $f$ and $z \mapsto \frac{1}{2} z$ as in item 2 of the claim.

Proof of Claim 6. Let $S$ be an attracting sector. We first notice that there exists an arc $\alpha$ included in the boundary of $S$, whose interior $\left({ }^{2}\right) \operatorname{Int}(\alpha)$ contains the fixed point 0 , and such that $f(\alpha) \subset \operatorname{Int}(\alpha)$. Indeed, consider a one-to-one continuous map $\varphi:(-1,1) \rightarrow \partial S$ such that $\varphi(0)=0$, and let $\hat{f}=$ $\varphi^{-1} f \varphi$. Since $f(S) \stackrel{0}{=} S$, this map $\hat{f}$ is well defined on some neighbourhood $V$ of 0 in $(-1,1)$, it is a homeomorphism from $V$ to $\hat{f}(V) \subset(-1,1)$, and it fixes 0 . Since $f$ preserves the orientation, and $f(S) \stackrel{0}{=} S, \hat{f}$ also preserves the orientation. Furthermore, for every positive $x \in V$, we have $\hat{f}(x)<x$ : otherwise the sequence $\left(\hat{f}^{-n}(x)\right)_{n \geq 0}$ would be well defined and included in $V$, and then $\varphi(x)$ would be included in $f^{n}(\partial S)$ for every positive $n$, contradicting the hypothesis that $\left(f^{n}(S)\right)_{n \geq 0}$ converges to 0 . Similarly we get $\hat{f}(x)>x$ for every negative $x$, so $\hat{f}(\operatorname{Clos}(V)) \subset V$. Finally, the arc $\alpha$ is obtained as $\varphi(\operatorname{Clos}(V))$.

Consider the set

$$
A:=\bigcup_{n \geq 0} f^{-n}(\alpha)
$$

This is clearly a continuous one-to-one image of the real line, and $f(A)=A$. By definition of an attracting sector, there exists an integer $n_{0}$ such that for every $n \geq n_{0}, f^{n}(S)$ is disjoint from the compact set $f^{-1}(\alpha) \backslash \operatorname{Int}(\alpha)$. Then $A \cap S=f^{-n_{0}}(\alpha) \cap S$. In particular, we can find a simple arc $\beta$ such that $\alpha \cup \beta$ is a Jordan curve included in $S$, and whose intersection with $A$ reduces to $\alpha$.

Let $D_{0}$ be the topological closed disc bounded by $\alpha \cup \beta$. Then $D_{0}$ is included in $S$ and coincides with $S$ in a neighbourhood of 0 , and $D_{0} \cap A=\alpha$. Note that for every $n, f^{n}\left(D_{0}\right) \cap A=f^{n}\left(D_{0} \cap A\right)=f^{n}(\alpha)$. The disc $D_{0}$ is clearly an attracting sector. Let $n_{0}$ be a positive integer such that for every $n \geq n_{0}, f^{n}\left(D_{0}\right)$ does not meet $\beta$. Thus $f^{n}\left(D_{0}\right) \subset \operatorname{Int}\left(D_{0}\right) \cup f^{n}(\alpha)$.

We can now find a closed topological disc $S^{\prime} \subset D_{0}$, having the same germ at 0 as $D_{0}$, which is a nice attracting sector for $f$. For this we can make a construction similar to the proof of Proposition 4, with the following adaptations. Now we choose the topological discs $D_{i}$ having the same germ at 0 , containing $\alpha$, and such that $\operatorname{Int}\left(D_{i}\right) \cup \alpha \supset D_{i+1}$ and $\operatorname{Int}\left(D_{n-1}\right) \cup f^{n}(\alpha)$

$\left({ }^{2}\right)$ The interior of a curve is defined to be the curve minus its endpoints. 
$\supset f^{n}\left(D_{0}\right)$. As before, we consider the set

$$
O=\operatorname{Int}\left(D_{n-1}\right) \cap \operatorname{Int}\left(f\left(D_{n-2}\right)\right) \cap \cdots \cap \operatorname{Int}\left(f^{n-1}\left(D_{0}\right)\right) .
$$

Now the open set $U$ is defined to be the unique connected component of $O$ that has the same germ at 0 as the $D_{i}$ 's, and $S^{\prime}$ is the closure of $U$. Then $f\left(S^{\prime}\right) \subset U \cup f^{n}(\alpha)$, and $f^{n}\left(S^{\prime}\right)$ contains $f^{n}(\alpha)$; and thus $S^{\prime} \backslash f\left(S^{\prime}\right)$ is connected. The details are left to the reader.

The proof of item 2 is straightforward by using the fundamental domain $\operatorname{Clos}\left(S^{\prime} \backslash f\left(S^{\prime}\right)\right)$.

2.2. Regular invariant petals. We first recall a theorem of Kerékjártó ([Ke34b]).

THEOREM 8 (Kerékjártó). Let $f$ be a homeomorphism of the plane that preserves the orientation, and suppose that for any compact set $K$ the orbit $\left(f^{n}(K)\right)_{n \geq 0}$ converges to the point at infinity in the sphere $\mathbb{R}^{2} \cup\{\infty\}$. Then $f$ is topologically conjugate to the translation $z \mapsto z+1$.

Sketch of proof. By considering the space of orbits, the problem can be brought into the realm of the classification of surfaces; then the conclusion follows from the fact that any (Hausdorff) surface without boundary, whose fundamental group is the group of integers, is homeomorphic to an infinite cylinder (see for example [AS]).

Definition 9. An invariant petal for $f$ is a topological closed disc $P$ whose boundary contains the fixed point 0 , and such that $f(P)=P$. An invariant petal is called regular if for every compact set $K \subset P \backslash\{0\}$, the sequence $\left(f^{n}(K)\right)_{n \geq 0}$ converges to 0 .

REMARK 10. If $P$ is a regular invariant petal then $f$ has no fixed point on the topological line $\partial P \backslash\{0\}$. Thus we may endow this line with a dynamical order such that $f(x)>x$ for any $x \neq 0$ on $\partial P$. The petal will be called direct if this dynamical order is compatible with the topological (usual) orientation of $\partial P$ as a Jordan curve of the oriented plane (for which the interior of $P$ is "on the left" of $\partial P$ ); in the opposite case it will be called indirect.

An adaptation of the proof of Kerékjártó's theorem yields the following.

Claim 11. Let $P$ be a regular invariant petal for $f \in \mathcal{H}^{+}$. If $P$ is direct then the restriction $f_{\mid P}$ is topologically conjugate, via an orientation preserving homeomorphism, to the restriction of the translation $z \mapsto z+1$ to the closed half-sphere $\{x+i y: y \geq 0\} \cup\{\infty\}$ of the Riemann sphere $\hat{\mathbb{C}}$. The same is true if $P$ is indirect with $z+1$ replaced by $z-1$.

2.3. Characterization of parabolic homeomorphisms. We can now characterize the local dynamics of parabolic homeomorphisms. For any set $D$ the maximal invariant subset of $D$ is the set $\bigcap_{n \in \mathbb{Z}} f^{n}(D)$ of points whose whole orbit is included in $D$. 
Proposition 12 (see Figure 1). Let $f \in \mathcal{H}^{+}$. Fix some integer $l \geq 1$. Then $f$ is locally topologically conjugate to $z \mapsto z\left(1+z^{l}\right)$ if and only if there exists a neighbourhood of 0 which is a topological closed disc D, called a nice disc, such that

1. the maximal invariant subset of $D$ is the union of $2 l$ regular invariant petals $P_{1}, \ldots, P_{2 l}$ whose pairwise intersections are $\{0\}$;

2. the sets $\partial D \cap P_{i}$ are connected, and the cyclic order of these sets along $\partial D$ coincides with the order of the indices $i \in \mathbb{Z} / 2 l \mathbb{Z}$;

3. for every $i$, let $S_{i}$ be the closure of the connected component of $D \backslash\left(P_{1} \cup \cdots \cup P_{2 l}\right)$ meeting both $P_{i}$ and $P_{i+1}$; then $S_{i}$ is a nice attracting sector for $i$ odd and a nice repulsive sector for $i$ even.

The next statement takes into account a possible permutation of the petals.

Proposition 13. Let $f \in \mathcal{H}^{+}$and suppose that for some positive integer $n_{0}$ the map $f^{n_{0}}$ is locally topologically conjugate to a parabolic homeomorphism. Then so is $f$.

The proofs are delayed until Section 4.

3. Proof of the theorem. From now on, $f$ denotes an orientation preserving homeomorphism of the plane that fixes 0 and has the short trip property. For every set $V$ we define the sets

$$
W^{\mathrm{s}}(V)=\bigcap_{n \geq 0} f^{-n}(V) \text { and } W^{\mathrm{u}}(V)=\bigcap_{n \leq 0} f^{-n}(V) \text {. }
$$

We fix some open neighbourhood $V$ of 0 as in the definition of the short trip property. Since our hypothesis is symmetric in time, both the above sets share the same properties, and we will usually restrict the study to $W^{\mathrm{s}}(V)$.

3.1. Orbits. The following lemma shows in particular that the orbits of points near 0 can only converge to 0 or escape from the neighbourhood $V$. Note that this lemma still holds in any dimension.

LEMMA 14.

1. For every compact subset $K$ of $W^{\mathrm{s}}(V) \backslash\{0\}$, the sequence $\left(f^{n}(K)\right)_{n \geq 0}$ converges to 0 .

2. The set $W^{\mathrm{s}}(V) \backslash\{0\}$ is open.

3. The set $W^{\mathrm{s}}(V) \cup W^{\mathrm{u}}(V)$ is a neighbourhood of 0 .

4. If $W^{\mathrm{s}}(V)$ is a neighbourhood of 0 then $f$ is locally topologically conjugate to $z \mapsto \frac{1}{2} z$; if $W^{\mathrm{u}}(V)$ is a neighbourhood of 0 then $f$ is locally topologically conjugate to $z \mapsto 2 z$.

Proof. Let $K$ be a compact subset of $W^{\mathrm{s}}(V) \backslash\{0\}$, and let $W$ be some neighbourhood of 0 disjoint from $K$. Note that by definition of $W^{\mathrm{s}}(V)$, for 
every positive $n, f^{n}(K) \subset V$. Now let $N_{W}$ be given by the short trip property. Then the property forces $f^{n}(K) \subset W$ for every $n>N_{W}$. This proves item 1 of the lemma.

Let $x \neq 0$ be some point in $W^{\mathrm{s}}(V)$, and $W$ a neighbourhood of 0 whose closure does not contain $x$, and such that $W \cup f(W) \subset V$. Let $N_{W}$ be given by the short trip property. Let

$$
O=\left(\bigcap_{n=0}^{N_{W}} f^{-n}(V)\right) \backslash \operatorname{Clos}(W) .
$$

This is an open set that contains $x$. We prove item 2 of the lemma by showing that $O \subset W^{\mathrm{s}}(V)$. To see this, let $y \in O$. By definition of $N_{W}$ in the short trip property we have $f^{N_{W}}(y) \in W$. Then we claim that $f^{n}(y) \in W$ for every $n \geq N_{W}$, which will entail $y \in W^{\mathrm{s}}(V)$ as desired. Assume otherwise and let $n_{0}$ be the least integer after $N_{W}$ such that $f^{n_{0}}(y) \notin W$. Since $f(W) \subset V$ we have $f^{n_{0}}(y) \in V$. The segment of orbit $y, \ldots, f^{n_{0}}(y)$ contradicts the definition of $N_{W}$. This completes the proof of item 2 .

We consider again a neighbourhood $W$ of 0 such that $W \cup f(W) \cup$ $f^{-1}(W) \subset V$ and $N_{W}$ given by the short trip property. We define the following neighbourhood of 0 :

$$
Z=\bigcap_{n=-N_{W}}^{N_{W}} f^{-n}(W) .
$$

We prove by contradiction that $Z \subset W^{\mathrm{s}}(V) \cup W^{\mathrm{u}}(V)$. Assume some $x \in Z$ belongs neither to $W^{\mathrm{s}}(V)$ nor to $W^{\mathrm{u}}(V)$. Since $W \subset V$, the orbit $\left(f^{n}(x)\right)$ of $x$ leaves $W$ both in the past and in the future; but by definition of $Z$ this cannot happen for $n$ between $-N_{W}$ and $N_{W}$. Let $r, s$ be the least positive integers such that $f^{-r}(x)$ and $f^{s}(x)$ do not belong to $W$; since $f(W) \cup f^{-1}(W) \subset V$, both points belong to $V \backslash W$ and again we have found a segment of orbit of length $r+s>2 N_{W}$ contradicting the definition of $N_{W}$.

Finally, we notice that item 4 is a consequence of item 1 and the topological characterization of contractions (Proposition 4 above).

3.2. Construction of the petals. We still consider a homeomorphism $f \in \mathcal{H}^{+}$with the short trip property, and from now on we assume that $f$ is locally conjugate neither to the contraction $z \mapsto \frac{1}{2} z$ nor to the dilatation $z \mapsto 2 z$. We aim to prove that $f$ is locally conjugate to a parabolic homeomorphism by ultimately applying Propositions 12 and 13. The main task will be to construct the family of periodic petals. As a first approximation we will select a finite number of connected components of $W^{\mathrm{s}}(V) \cap W^{\mathrm{u}}(V) \backslash\{0\}$, hoping to find one petal inside each of these components.

We fix an open neighbourhood $V$ of 0 as before, and we assume $V$ is simply connected. According to item 3 of the previous lemma, we can 
choose a topological closed disc $D$ which is a neighbourhood of 0 included in $W^{\mathrm{s}}(V) \cup W^{\mathrm{u}}(V)$. According to item 4, since we excluded the cases of contractions and dilatations, $D$ is included neither in $W^{\mathrm{s}}(V)$ nor in $W^{\mathrm{u}}(V)$. By compactness we can decompose $\partial D$ into the concatenation of $2 l \geq 2$ arcs $\alpha_{1}, \ldots, \alpha_{2 l}$ such that $\alpha_{i}$ is included in $W^{\mathrm{s}}(V)$ for $i$ odd and in $W^{\mathrm{u}}(V)$ for $i$ even. We make the following minimality hypothesis: the number $l$ is minimal among all such choices of topological closed discs $D$ and decompositions of $\partial D$.

For every $i$ (integer modulo $2 l$ ) the common endpoint $x_{i}$ of $\alpha_{i-1}$ and $\alpha_{i}$ belongs to $W^{\mathrm{s}}(V) \cap W^{\mathrm{u}}(V)$. We denote by $\mathcal{C}_{i}$ the connected component of $W^{\mathrm{s}}(V) \cap W^{\mathrm{u}}(V) \backslash\{0\}$ that contains $x_{i}$. According to item 2 of Lemma 14, $\mathcal{C}_{i}$ is open. Let $D^{\prime}$ be a topological closed disc; since $V$ is simply connected, if $\partial D^{\prime} \subset V$ then $D^{\prime} \subset V$. Applying this to the iterates of $D^{\prime}$, we see that if $\partial D^{\prime} \subset W^{\mathrm{s}}(V) \cap W^{\mathrm{u}}(V)$, then $D^{\prime} \subset W^{\mathrm{s}}(V) \cap W^{\mathrm{u}}(V)$. Since $W^{\mathrm{s}}(V)$ is not a neighbourhood of 0 , we get the following consequence.

Lemma 15. Any connected component of $W^{\mathrm{s}}(V) \cap W^{\mathrm{u}}(V) \backslash\{0\}$ is open and simply connected. In particular, the sets $\mathcal{C}_{i}$ are homeomorphic to the plane. Furthermore, any topological closed disc $D$ whose boundary is included in $\mathcal{C}_{i} \cup\{0\}$ is also included in $\mathcal{C}_{i} \cup\{0\}$.

The next lemma is the fundamental step in the construction of the periodic petals. No dynamics is involved here; indeed, we will only need properties 2 and 3 from Lemma 14 on the topology of $W^{\mathrm{s}}(V)$ and $W^{\mathrm{u}}(V)$.

Lemma 16. For every $i$, the closure of $\mathcal{C}_{i}$ contains the fixed point 0.

Proof. For notational simplicity we assume $i=1$, and we write $\mathcal{C}=\mathcal{C}_{1}$ and $x=x_{1} \in \alpha_{2 l} \cap \alpha_{1}$. Using the Schoenflies theorem, up to a change of coordinates, we can assume that $D$ is a euclidean closed disc.

We will argue by contradiction. Assuming that 0 does not belong to the closure of $\mathcal{C}$, we will construct a simple arc $\alpha$ with the following properties (we denote by $\partial \alpha$ the set of endpoints of $\alpha$ and set $\operatorname{Int}(\alpha)=\alpha \backslash \partial \alpha$ ):

1. $\operatorname{Int}(\alpha) \subset \operatorname{Int}(D), \partial \alpha \subset \partial D$;

2. $\alpha$ separates $\left({ }^{3}\right) x$ from 0 in $D$;

3. either $\alpha \subset W^{\mathrm{s}}(V)$ and $\partial \alpha \cap W^{\mathrm{u}}(V)=\emptyset$, or $\alpha \subset W^{\mathrm{u}}(V)$ and $\partial \alpha \cap$ $W^{\mathrm{s}}(V)=\emptyset$.

From this we will get a contradiction as follows (see Figure 3(a)). Assume for example that the first case of the last item holds. Let $1 \leq i_{1} \leq i_{2} \leq 2 l$ be such that the endpoints of $\alpha$ are respectively in $\alpha_{i_{1}}$ and $\alpha_{i_{2}}$. Since $\partial \alpha$ does not meet $W^{\mathrm{u}}(V)$, both $i_{1}$ and $i_{2}$ are odd, and in particular $1 \leq i_{1} \leq i_{2}<2 l$.

$\left({ }^{3}\right)$ A set $A$ separates two points in a set $B$ if the two points belong to distinct connected components of $B \backslash A$. 


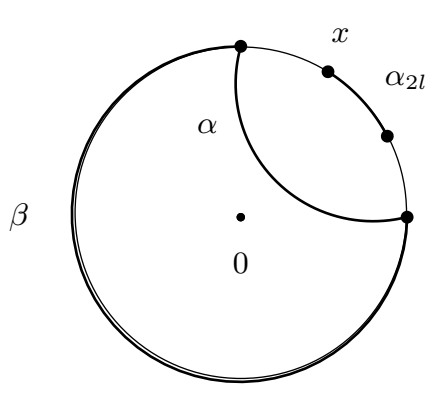

(a) The arc $\alpha$

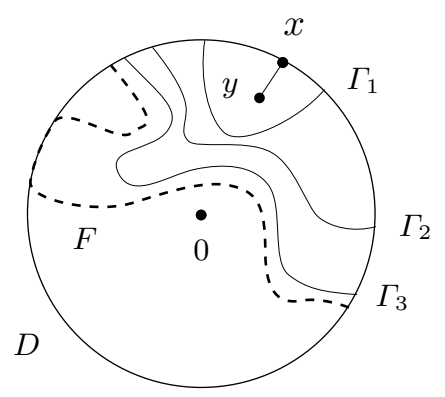

(b) The sequence $\Gamma_{k}$ and the limit set $F$

Fig. 3. Proof of Lemma 16

Let $\beta \subset \partial D$ be the arc with the same endpoints as $\alpha$ and not containing $x$; then $\beta$ is covered by $\alpha_{i_{1}} \cup \cdots \cup \alpha_{i_{2}}$, and from the second point we see that the Jordan curve $\alpha \cup \beta$ surrounds 0. Since $\alpha \cup \alpha_{i_{1}} \cup \alpha_{i_{2}} \subset W^{\mathrm{s}}(V)$, we can write $\alpha \cup \beta$ as the concatenation of $i_{2}-i_{1}$ arcs, each included in $W^{\mathrm{s}}(V)$ or $W^{\mathrm{u}}(V)$. This contradicts the minimality hypothesis on $l$ since $i_{2}-i_{1}<2 l$.

We now assume that $0 \notin \operatorname{Clos}(\mathcal{C})$ and turn to the construction of such an arc $\alpha$. According to Lemma 15, there exists a homeomorphism $\Phi: \mathbb{R}^{2} \rightarrow \mathcal{C}$. Let $\left(D_{k}\right)$ be the sequence of images under $\Phi$ of the concentric discs with radius $k$ and centre $\Phi^{-1}(x)$. Thus:

- $x \in D_{1}$,

- $D_{k} \subset \operatorname{Int}\left(D_{k+1}\right)$,

- $\bigcup_{k \geq 0} D_{k}=\mathcal{C}$.

Let $y$ be some point in $\operatorname{Int}(D)$ sufficiently near $x$ so that the segment $[x y]$ is included in $D_{1} \cap D$. For every $k$, the set $\partial D_{k} \cap \operatorname{Int}(D)$ is closed in $\operatorname{Int}(D)$ and separates $y$ from 0 in $\operatorname{Int}(D)$ since $0 \notin D_{k}$. According to Theorem V.14.3 in [New], there exists a connected component of $\partial D_{k} \cap \operatorname{Int}(D)$ that also separates $y$ from 0 . Let $\Gamma_{k}$ denotes the closure of this component; thus $\Gamma_{k}$ is a subarc of the Jordan curve $\partial D_{k}$ with $\operatorname{Int}\left(\Gamma_{k}\right) \subset \operatorname{Int}(D), \partial \Gamma_{k} \subset \partial D$, and it separates $x$ from 0 in $D$; in other words, it satisfies the first two properties above required for the arc $\alpha$.

Remember that the space of compact connected subsets of $D$ is compact under the Hausdorff metric. Thus, up to taking subsequences, we can assume that $\left(\Gamma_{k}\right)$ converges to a compact connected set $F \subset D$ (see Figure 3(b)). Since $\Gamma_{k} \subset \partial D_{k}$, the set $F$ is included in $\partial \mathcal{C}$. By assumption, $\partial \mathcal{C}$ does not contain 0 , so neither does $F$. Then again $F$ separates $y$ from 0 in $\operatorname{Int}(D)$ : if not, there would exist an arc in $\operatorname{Int}(D)$ from $y$ to 0 missing $F$, but then this arc would also miss $\Gamma_{k}$ for sufficiently large $k$, contrary to the property that $\Gamma_{k}$ separates $y$ from 0 . 
Since $\mathcal{C}$ is a connected component of the open set $W^{\mathrm{s}}(V) \cap W^{\mathrm{u}}(V) \backslash\{0\}$, its boundary $\partial \mathcal{C}$ is disjoint from this set. Thus $F$ is disjoint from $W^{\mathrm{s}}(V) \cap$ $W^{\mathrm{u}}(V)$; and since it is included in $D$ it is covered by the two open sets $W^{\mathrm{s}}(V) \backslash\{0\}$ and $W^{\mathrm{u}}(V) \backslash\{0\}$. Since $F$ is connected, it must be included in one of these sets, and disjoint from the other.

To fix ideas, suppose that $F \subset W^{\mathrm{s}}(V) \backslash W^{\mathrm{u}}(V)$. The arc $\Gamma_{k}$ is included in $W^{\mathrm{s}}(V)$. Now this arc almost satisfies the three properties above required for the arc $\alpha$ : it only fails to have its endpoints outside $W^{\mathrm{u}}(V)$. To remedy this we notice that, up to taking sequences, the two sequences of endpoints $\left(\Gamma_{k}(0)\right),\left(\Gamma_{k}(1)\right)$ converge to some $z_{0}, z_{1} \in F \cap \partial D$. Since $F \subset W^{\mathrm{s}}(V) \backslash\{0\}$ we can choose $\varepsilon>0$ so that the euclidean balls $B_{0}, B_{1}$ of radius $\varepsilon$ and respective centres $z_{0}, z_{1}$ are included in $W^{\mathrm{s}}(V)$. For $k$ large enough, $\Gamma_{k}$ meets both balls, and then we construct the desired arc $\alpha$ by modifying $\Gamma_{k}$ near its endpoints: we replace two small extreme subarcs of $\Gamma_{k}$, respectively included in $B_{0}$ and $B_{1}$, by two euclidean segments reaching $z_{0}$ and $z_{1}$. Note that since $D$ is a euclidean disc, both segments, apart from their endpoints $z_{0}, z_{1}$, are included in $\operatorname{Int}(D)$. The endpoints $z_{0}, z_{1}$ of the resulting arc $\alpha$ are in $F$, thus outside $W^{\mathrm{u}}(V)$, and $\alpha$ has the third property while still satisfying the first two. As explained at the beginning of the proof, the existence of $\alpha$ contradicts the minimality of $l$.

3.3. Periodicity of the petals. Unfortunately, we are not able to prove directly that the sets $\mathcal{C}_{i}$ of the previous section are periodic for $f$. To overcome this difficulty we will consider slightly larger sets $\mathcal{C}_{i}^{\prime}$ which will turn out to be periodic. In the next section we will find a periodic petal inside each $\mathcal{C}_{i}^{\prime}$.

We suppose that the closure of $V$ is included in some neighbourhood $V^{\prime}$ of 0 which still has the short trip property. In other words, we apply the results of the previous sections with $V$ small enough to meet this new assumption. We note that Lemmas 14 and 15 apply to $V^{\prime}$. Obviously the inclusions $W^{\mathrm{s}}(V) \subset W^{\mathrm{s}}\left(V^{\prime}\right)$ and $W^{\mathrm{u}}(V) \subset W^{\mathrm{u}}\left(V^{\prime}\right)$ hold. Let the disc $D$ and the sets $\mathcal{C}_{i}$ be defined from $V$ as in the previous section. Each $\mathcal{C}_{i}$ is connected and included in $W^{\mathrm{s}}\left(V^{\prime}\right) \cap W^{\mathrm{u}}\left(V^{\prime}\right) \backslash\{0\}$, and thus included in a connected component of $W^{\mathrm{s}}\left(V^{\prime}\right) \cap W^{\mathrm{u}}\left(V^{\prime}\right) \backslash\{0\}$, which we denote by $\mathcal{C}_{i}^{\prime}$.

Lemma 17. The sets $\mathcal{C}_{i}^{\prime}$ are periodic: for every $i$ there exists some positive integer $q_{i}$ such that $f^{q_{i}}\left(\mathcal{C}_{i}^{\prime}\right)=\mathcal{C}_{i}^{\prime}$.

Proof. We first note that the set $W^{\mathrm{s}}\left(V^{\prime}\right) \cap W^{\mathrm{u}}\left(V^{\prime}\right) \backslash\{0\}$ is invariant under $f$, and hence for every $n, f^{n}\left(\mathcal{C}_{i}^{\prime}\right)$ is a connected component of that set.

We claim that for every $i$ there exist infinitely many $n$ such that $f^{n}\left(\mathcal{C}_{i}\right)$ meets the circle $\partial D$. Assuming the claim, we choose some point $x$, any neighbourhood of which contains points of $\partial D \cap f^{n_{k}}\left(\mathcal{C}_{i}\right)$ for infinitely many values 
of $n$. Since $x$ is a limit of points whose whole orbits are included in $V$, its orbit is included in $\operatorname{Clos}(V) \subset V^{\prime}$; in other words, $x \in W^{\mathrm{s}}\left(V^{\prime}\right) \cap W^{\mathrm{u}}\left(V^{\prime}\right) \backslash\{0\}$. Let $O$ be the connected component of this last set containing $x$. According to Lemma 15, $O$ is open, and thus there exist infinitely many integers $n$ such that $f^{n}\left(\mathcal{C}_{i}\right)$ meets $O$. For every such $n, f^{n}\left(\mathcal{C}_{i}^{\prime}\right)$ is a connected component of $W^{\mathrm{s}}\left(V^{\prime}\right) \cap W^{\mathrm{u}}\left(V^{\prime}\right) \backslash\{0\}$ that meets $O$, thus it coincides with $O$. Hence we can find two integers $n_{1}<n_{2}$ such that $f^{n_{1}}\left(\mathcal{C}_{i}^{\prime}\right)=f^{n_{2}}\left(\mathcal{C}_{i}^{\prime}\right)$, which proves that $\mathcal{C}_{i}^{\prime}$ is periodic.

We prove the claim. By Lemma 16 the fixed point 0 belongs to the closure of $\mathcal{C}_{i}$. Since $\mathcal{C}_{i} \cup\{0\}$ is not a neighbourhood of 0 , this point also belongs to the closure of $\partial \mathcal{C}_{i}$. Furthermore,

$$
\begin{aligned}
\left(\partial \mathcal{C}_{i}\right) \backslash\{0\} & \subset \partial\left(W^{\mathrm{s}}(V) \cap W^{\mathrm{u}}(V)\right) \backslash\{0\} \\
& \subset\left(W^{\mathrm{s}}(\operatorname{Clos} V) \cap W^{\mathrm{u}}(\operatorname{Clos} V)\right) \backslash\left(W^{\mathrm{s}}(V) \cap W^{\mathrm{u}}(V)\right) .
\end{aligned}
$$

Consequently, for any $z \in \partial \mathcal{C}_{i} \backslash\{0\}$ there exists an integer $n$ such that $f^{n}(z) \in \partial V$. Let $\left(z_{k}\right)$ be a sequence in $\partial \mathcal{C}_{i}$ converging to 0 . Then any sequence $n_{k}$ such that $f^{n_{k}}\left(z_{k}\right) \in \partial V$ is unbounded, because the union of finitely many iterates of $\partial V$ is a closed set which does not contain 0 . For any $k$ the set $f^{n_{k}}\left(\mathcal{C}_{i}\right)$ is connected, its closure contains 0 and meets $\partial V$, thus it also meets $\partial D$. This completes the proof of the claim.

3.4. Construction of the local conjugacy. We finally define the petals. According to the previous lemma we can choose some $n_{0}>0$ such that $F=f^{n_{0}}$ leaves invariant every set $\mathcal{C}_{i}^{\prime}$. In view of Proposition 13, Theorem 3 will follow from the fact that $F$ is locally conjugate to a locally holomorphic parabolic homeomorphism. Let us prove this fact.

Recall that $\mathcal{C}_{i}^{\prime}$ is homeomorphic to the plane (Lemma 15), and for any compact set $K \subset \mathcal{C}_{i}^{\prime}$, the sequences $\left(f^{n}(K)\right)_{n \geq 0}$ and $\left(f^{n}(K)\right)_{n \leq 0}$ converge to 0 (Lemma 14). Consequently, Theorem 8 tells us that for every $i$ the restriction of $F$ to the invariant set $\mathcal{C}_{i}^{\prime}$ is conjugate to the plane translation $\tau: z \mapsto z+1$. Observe that any point is on a horizontal line

$$
\Delta^{\prime}=\bigcup_{n \in \mathbb{Z}} \tau^{n}\left(\delta^{\prime}\right)
$$

with compact $\delta^{\prime}$ (a horizontal segment). Bringing this line back under the conjugacy, we see that any point $x_{i} \in \mathcal{C}_{i}^{\prime}$ is on a set $\Delta_{i} \subset \mathcal{C}_{i}^{\prime}$ which is the union of the iterates under $F$ of a compact set (an arc) included in $\mathcal{C}_{i}^{\prime}$. By Lemma $14, \Delta_{i} \cup\{0\}$ is a Jordan curve. Let $P_{i}$ be the closed topological disc bounded by this curve (here we use the Schoenflies theorem). The last sentence of Lemma 15 entails that $P_{i}$ is included in $\mathcal{C}_{i}^{\prime}$, and then Lemma 14 shows that $P_{i}$ is a regular invariant petal for $F$.

The curve $\alpha_{i}$ meets both petals $P_{i}$ and $P_{i+1}$. Furthermore for $i$ odd we have $\alpha_{i} \subset W^{\mathrm{s}}(V)$, so by Lemma $14,\left(F^{n}\left(\alpha_{i}\right)\right)_{n \geq 0}$ converges to 0 , and 
$\left(F^{n}\left(\alpha_{i}\right)\right)_{n \leq 0}$ converges to 0 for $i$ even. Thus the construction of a local conjugacy between $F$ and $z \mapsto z\left(1+z^{l}\right)$ now boils down to the following lemma.

Lemma 18 (see Figure 1). Let $f \in \mathcal{H}^{+}$. Fix some integer $l \geq 1$. Assume the following hypotheses.

1. There exist $2 l$ regular invariant petals $P_{1}, \ldots, P_{2 l}$ whose pairwise intersections are $\{0\}$.

2. There exists a topological closed disc D which is a neighbourhood of 0 , and whose boundary is the concatenation of $2 l$ arcs $\alpha_{1}, \ldots, \alpha_{2 l}$, each having one endpoint on $P_{i}$ and the other on $P_{i+1}$.

3. For $i$ odd the sequence $\left(f^{n}\left(\alpha_{i}\right)\right)_{n \geq 0}$ converges to 0 , and for $i$ even the sequence $\left(f^{n}\left(\alpha_{i}\right)\right)_{n \leq 0}$ converges to 0 .

Then $f$ is locally conjugate to $z \mapsto z\left(1+z^{l}\right)$.

Note that we do not suppose that $\partial D \cap P_{i}$ is connected, nor that $\alpha_{i}$ does not meet some petal $P_{j}$ with $j \neq i, i+1$, in contrast to Proposition 12 . An important step of the proof will be to check that the petal indexation coincides with their cyclic order around 0.

Proof of Lemma 18. Consider a homeomorphism $f \in \mathcal{H}^{+}$satisfying the hypotheses of the lemma. The arc $\alpha_{i}$ contains a minimal subarc $\alpha_{i}^{\prime}$ connecting $P_{i}$ to $P_{i+1}$; the endpoints of $\alpha_{i}^{\prime}$ are respectively on $P_{i}$ and $P_{i+1}$, and its interior $\operatorname{Int}\left(\alpha_{i}^{\prime}\right)$ is disjoint from $P_{i}$ and $P_{i+1}$. Let $i$ be odd, so that $\left(f^{n}\left(\alpha_{i}^{\prime}\right)\right)_{n \geq 0}$ converges to 0 . Then we define an attracting sector $S_{i}^{\prime}$ as follows. We consider the curve obtained by concatenating the arc $\alpha_{i}^{\prime}$, the subarc of $\partial P_{i}$ from the endpoint of $\alpha$ to 0 following the dynamical orientation of $\partial P_{i}$, and the similar subarc on $P_{i+1}$ (see Remark 10). This is a Jordan curve, it bounds a topological closed disc $S_{i}^{\prime}$. Clearly $\left(f^{n}\left(\partial S_{i}^{\prime}\right)\right)_{n \geq 0}$ converges to 0 , and thus so does $\left(f^{n}\left(S_{i}^{\prime}\right)\right)_{n \geq 0}$; in other words, $S_{i}^{\prime}$ is indeed an attracting sector. Note that as a consequence, it can contain neither $P_{i}$ nor $P_{i+1}$, because an attracting sector contains no invariant set. We apply item 1 of Claim 6 to get a nice attracting sector $S_{i} \subset S_{i}^{\prime}$ having the same germ as $S_{i}^{\prime}$ at 0 . For $i$ even we symmetrically define a repulsive sector $S_{i}^{\prime}$ and a nice repulsive sector $S_{i}$.

Since the petals are topological closed discs whose pairwise intersection is $\{0\}$, the Schoenflies theorem shows that the union of the petals is homeomorphic to the model pictured on the left of Figure 1; but we still have to prove that their cyclic order is as shown on the right of the figure (or the reverse one). For this we argue by contradiction. Suppose there exists some $i$ such that the petals $P_{i}$ and $P_{i+1}$ are not adjacent: they are locally separated near 0 by the union of the other petals. Then there exists another petal $P_{j}$ such that the sector $S_{i}$ contains a neighbourhood of 0 in $P_{j}$; in other words, $\operatorname{Clos}\left(P_{j} \backslash S_{i}\right)$ is a compact subset of $P_{j} \backslash\{0\}$. Using Claim 11 that 
describes the dynamics of $f$ on $P_{j}$, we find a point $x \neq 0$ whose full orbit $\left\{f^{n}(x): n \in \mathbb{Z}\right\}$ is included in $P_{j} \cap S_{i}$. But an attracting or repulsive sector contains no full orbit, which provides the desired contradiction.

Up to reversing the indexation, we may now assume that the petals are indexed in the positive cyclic order around 0 (so that the Schoenflies theorem provides an orientation preserving homeomorphism that sends each $P_{i}$ onto the model of Figure 1). Since $S_{i}$ contains neither $P_{i}$ nor $P_{i+1}$, the dynamical order on the boundaries of the petals is as indicated in Figure 1: the petal $P_{i}$ is direct for $i$ odd and indirect for $i$ even.

Up to replacing $S_{i}$ with some smaller nice sector, we deduce that

1. for any $i, j$ with $j \neq i, i+1$, we have $P_{i} \cap S_{j}=\{0\}$;

2. for any $i \neq j$, we have $S_{i} \cap S_{j}=\{0\}=f^{-1}\left(S_{i}\right) \cap S_{j}$.

Consider the set $D=P_{1} \cup S_{1} \cup \cdots \cup P_{2 l} \cup S_{2 l}$. Thanks to item 2 the maximal invariant subset of $D$ is the union of the petals $P_{i}$. Thus $D$ is a topological closed disc satisfying the hypotheses of Proposition 12. Now the lemma follows from the proposition.

\section{Proof of Propositions 12 and $\mathbf{1 3}$}

Proof of Proposition 12. The fact that for the map $z \mapsto z\left(1+z^{l}\right)$ there exists a topological closed disc $D$ with properties $1-3$ of the proposition is part of the proof of the Camacho-Leau-Fatou theorem (see [Cam, Mil]).

We turn to the proof of the reverse implication. We consider a homeomorphism $f \in \mathcal{H}^{+}$and a disc $D$ with properties $1-3$ of the proposition. We have to prove that if $f^{\prime} \in \mathcal{H}^{+}$and a disc $D^{\prime}$ has the same properties (with the same $l$ ) then $f$ and $f^{\prime}$ are locally topologically conjugate. Note that the union of all sectors $S_{i}$ and petals $P_{i}$ is equal to $D$.

Let $i$ be an odd integer. Since $S_{i}$ is an attracting sector between $P_{i}$ and $P_{i+1}$, the petal $P_{i}$ is direct, while $P_{i+1}$ is indirect (see Remark 10). The same is true for $f^{\prime}$. Thus according to Claim 11, the restrictions of $f$ and $f^{\prime}$ to $P_{i}$ and $P_{i}^{\prime}$ are conjugate. The conjugacies can be glued together to obtain an orientation preserving homeomorphism $\Phi: \bigcup P_{i} \rightarrow \bigcup P_{i}^{\prime}$ which sends $P_{i}$ onto $P_{i}^{\prime}$ and is a conjugacy between the restrictions of $f$ and $f^{\prime}$.

The image under $\Phi$ of $S_{i} \cap\left(P_{i} \cup P_{i+1}\right)$ is not necessarily equal to $S_{i}^{\prime} \cap\left(P_{i}^{\prime} \cup\right.$ $\left.P_{i+1}^{\prime}\right)$. But using item 2 of Claim 6 we can replace $S_{i}$ and $S_{i}^{\prime}$ with smaller nice attracting sectors so that this equality becomes true (see item 2 of Remark 7 ). We can now use item 3 of Remark 7 to extend $\Phi$ to a homeomorphism between $D$ and $D^{\prime}$, sending $S_{i}$ onto $S_{i}^{\prime}$ and conjugating the restrictions of $f$ and $f^{\prime}$. We do this for every attracting or repulsive sector $S_{i}$. We further extend $\Phi$ to a homeomorphism of the plane. The conjugacy relation $f^{\prime} \Phi=\Phi f$ is satisfied on $D \cap f^{-1}(D)$. This completes the proof of the proposition. 
To prove Proposition 13 we need a claim.

Claim 19. Let $Q_{1}, Q_{2}$ be two invariant petals included in a regular invariant petal $P$ for $F \in \mathcal{H}^{+}$. Then $Q_{1}$ meets $Q_{2}$, and there exists a unique connected component $O$ of $\operatorname{Int}\left(Q_{1}\right) \cap \operatorname{Int}\left(Q_{2}\right)$ such that $F(O)=O$. Furthermore, the closure of $O$ is a regular invariant petal for $F$.

Proof. The first part is easily proved using the translation model given by Claim 11. The only difficulty in the second part is to check that the closure of $O$ is indeed a topological closed disc. But this follows from the previously quoted result of Kerékjártó ([Ke23, LCY]).

Also note that if $Q \subset P$ are two regular invariant petals and $P$ is direct then $Q$ is direct.

Proof of Proposition 13. Let $f^{n_{0}}=F$ be conjugate to a parabolic homeomorphism $F_{0}$. Up to increasing $n_{0}$, we can assume that $F_{0}^{\prime}(0)=1$, and thus $F$ is locally conjugate to $z \mapsto z\left(1+z^{l}\right)$ for some integer $l$. Let $D$ be a nice disc for $F$, and let $\left\{P_{1}, \ldots, P_{2 l}\right\}$ be the family of petals associated with $D$, as given by Proposition 12 .

For each $i$ we choose a small invariant petal $Q_{i}$ for $F$ included in $P_{i}$. Since $f^{n_{0}}=F$ and $Q_{i}$ is invariant for $F$, if $Q_{i}$ is small enough then every iterate $f^{n}\left(Q_{i}\right)$ is included in $D$. Since $f^{n}\left(Q_{i}\right) \backslash\{0\}$ is connected and invariant for $F$, it is included in a connected component of the $F$-maximal invariant subset of $D \backslash\{0\}$, that is, $f^{n}\left(Q_{i}\right)$ is included in some petal $P_{j}$. Fix $j$ and consider the finite family of all the petals $f^{n}\left(Q_{i}\right)$ for $n \in \mathbb{Z}$ and $i \in \mathbb{Z} / 2 l \mathbb{Z}$ which are included in $P_{j}$. We denote the intersection of their interiors by $O_{j}$. Applying Claim 19 inductively we see that the closure of $O_{j}$ is a regular invariant petal for $F$; let us call it $\bar{P}_{j}$.

By construction the petals in the family $\left\{\bar{P}_{j}\right\}$ are permuted by $f$, their pairwise intersections are $\{0\}$, and their cyclic order around 0 is given by the cyclic order on the indices $j \in \mathbb{Z} / 2 l \mathbb{Z}$. Since $f$ is an orientation preserving homeomorphism, there exists $i_{0}$ such that $f\left(\bar{P}_{i}\right)=\bar{P}_{i+i_{0}}$ for every $i$. Furthermore, since $f$ respects the dynamical orders induced by $F$ on the boundary of the petals, $i_{0}$ must be even. The order $n_{0}^{\prime}$ of the permutation $i \mapsto i+i_{0}$ is a divisor of $n_{0}$ (maybe proper). It is easy to see that there exists another nice disc $\bar{D}$ for $F$ whose maximal invariant subset is the union of this family of petals. The nice attractive and repulsive sectors $\bar{S}_{i}$ for $F$, associated with $\bar{D}$, are clearly also attractive or repulsive sectors for $f^{n_{0}^{\prime}}$, and according to Claim 6 we can find within each $\bar{S}_{i}$ a nice attracting or repulsive sector $\overline{\bar{S}}_{i}$ for $f^{n_{0}^{\prime}}$ having the same germ at 0 . Now the topological closed disc $\overline{\bar{D}}$ defined as the union of all petals $\bar{P}_{i}$ and sectors $\overline{\bar{S}}_{i}$ is a nice disc for $f^{n_{0}^{\prime}}$, the hypotheses of Proposition 12 are satisfied, and $f^{n_{0}^{\prime}}$ is conjugate to $F$. 
Using these families of petals and sectors we are now in a position to construct a local conjugacy $\Phi$ between $f$ and the model map $f_{0}: z \mapsto$ $e^{2 i \pi i_{0} / 2 l} z\left(1+z^{l}\right)$. Note that $f_{0}^{n_{0}^{\prime}}$ is conjugate to $z \mapsto z\left(1+z^{l}\right)$ and that $f_{0}$ permutes a family of regular invariant petals for $f_{0}^{n_{0}^{\prime}}$. The construction of the conjugacy is similar to the one defined in the proof of Proposition 12. Here is the main difference: since the petals are permuted by $f$, we have first to define a conjugacy $\Phi$ between $f^{n_{0}^{\prime}}$ and $f_{0}^{n_{0}^{\prime}}$ on some petal $\bar{P}_{i}$, and then there is a unique way to extend it to the $f$-orbit of $\bar{P}_{i}$ to get a conjugacy between $f$ and $f_{0}$. We do the same for every $f$-orbit of petals, and for every $f$-orbit of a sector. This completes the proof of Proposition 13.

\section{References}

[AS] L. V. Ahlfors and L. Sario, Riemann Surfaces, Princeton Math. Ser. 26, Princeton Univ. Press, Princeton, NJ, 1960.

[BLR] F. Béguin et F. Le Roux, Ensemble oscillant d'un homéomorphisme de Brouwer, homéomorphismes de Reeb, Bull. Soc. Math. France 131 (2003), 149-210.

[BK] C. Bonatti and B. Kolev, Surface homeomorphisms with zero-dimensional singular set, Topology Appl. 90 (1998), 69-95.

[Cam] C. Camacho, On the local structure of conformal mappings and holomorphic vector fields, Astérisque 59-60 (1978), 83-94.

[GLP] J.-M. Gambaudo, P. Le Calvez et É. Pécou, Une généralisation d'un théorème de Naishul, C. R. Acad. Sci. Paris Sér. I Math. 323 (1996), 397-402.

[GP] J.-M. Gambaudo and É. Pécou, A topological invariant for volume preserving diffeomorphisms, Ergodic Theory Dynam. Systems 15 (1995), 535-541.

[Ham] O. H. Hamilton, A short proof of the Cartwright-Littlewood theorem, Canad. J. Math. 6 (1954), 522-524.

[Hi] K. Hiraide, Expansive homeomorphisms of compact surfaces are pseudo-Anosov, Osaka J. Math. 27 (1990), 117-162.

[Ke23] B. Kerékjártó, Vorlesungen über Topologie (I), Springer, Berlin, 1923.

[Ke34a] - Sur le caractère topologique des représentations conformes, C. R. Acad. Sci. Paris 198 (1934), 317-320.

[Ke34b] - Sur le groupe des transformations topologiques du plan, Ann. Scuola Norm. Sup. Pisa 3 (1934), 393-400.

[LCY] P. Le Calvez et J.-C. Yoccoz, Un théorème d'indice pour les homéomorphismes du plan au voisinage d'un point fixe, Ann. of Math. (2) 146 (1997), 241-293.

[Le] J. Lewowicz, Expansive homeomorphisms of surfaces, Bol. Soc. Brasil. Mat. (N.S.) 20 (1989), 113-133.

[LR1] F. Le Roux, Homéomorphismes de surfaces: théorèmes de la fleur de Leau-Fatou et de la variété stable, Astérisque 292 (2004).

[LR2] - L'ensemble de rotation d'un germe d'homéomorphisme de surface, work in progress.

[Mil] J. Milnor, Dynamics in One Complex Variable, Ann. of Math. Stud. 160, Princeton Univ. Press, Princeton, NJ, 2006.

[New] M. H. A. Newman, Elements of the Topology of Plane Sets of Points, Dover, New York, 1992. 
[Pé] É. Pécou, A topological invariant for nonlinear rotations of $\mathbb{R}^{3}$, Nonlinearity 10 (1997), 153-158.

Laboratoire de Mathématiques CNRS UMR 8628

Université Paris-Sud, Bât. 425

91405 Orsay Cedex, France

E-mail: frederic.le-roux@math.u-psud.fr

Received 25 July 2007;

in revised form 18 September 2007 